\title{
Evidence for subtypes of Freezing of gait in Parkinson's disease
}

Kaylena A. Ehgoetz Martens*, PhD, James M Shine, BSc/MBBS/PhD, Courtney C. Walton, PhD, Matthew J. Georgiades, BSc, Moran Gilat, MSc, Julie M. Hall, MSc, Alana J Muller, BMedSci, Jennifer YY Szeto, PhD, Simon JG Lewis, MBBCh/PhD Affiliation: Forefront, Brain and Mind Centre, University of Sydney 100 Mallet Street Camperdown 2050 New South Wales, Australia

${ }^{*}$ corresponding author:

kaylena.ehgoetzmartens@sydney.edu.au

1672 words; 150 word abstract

Running Title: Investigating subtypes of freezing

Search terms: Freezing of gait, Parkinson's disease, motor, cognitive, affective, limbic, heterogeneity

Study funding: KAEM is supported by a Parkinson Canada Fellowship; JMS is supported by a National Health and Medical Research Council CJ Martin Fellowship (1072403); JMH is supported by a Western Sydney University Postgraduate Research Award; Professor Lewis is supported by NHMRC-ARC Dementia Fellowship (1110414) and this work was supported by funding to Forefront, a collaborative research group dedicated to the study of non-Alzheimer disease degenerative dementias, from the National Health and Medical Research Council of

This is the author manuscript accepted for publication and has undergone full peer review but has not been through the copyediting, typesetting, pagination and proofreading process, which may lead to differences between this version and the Version of Record. Please cite this article as doi: $10.1002 / \mathrm{mds} .27417$

This article is protected by copyright. All rights reserved. 
Australia program grant (\#1037746 and \#1095127). MG, JYYS, CCW and AAM have no funding source to disclose.

Disclosures: Authors have no further disclosures

\begin{abstract}
Background: To identify and characterise subtypes of freezing of gait by utilizing a novel questionnaire designed to delineate freezing patterns based on self-reported and behavioural gait assessment.
\end{abstract}

Methods: 41 Parkinson's patients with freezing completed a 'Characterizing Freezing of Gait' questionnaire that identifies situations that exacerbate freezing. This instrument underwent examination for construct validity and internal consistency, after which a data-driven clustering approach was employed to identify distinct patterns amongst individual responses. Behavioural freezing assessments in both dopaminergic states were compared across three identified subgroups.

Results: This novel questionnaire demonstrated construct validity (severity scores correlated with percentage of time 'frozen'; $r=0.54$ ) and internal consistency (Cronbach's alpha $=0.937$, and thus demonstrated promising utility for identifying patterns of freezing that are independently related to motor, anxiety and attentional impairments.

Conclusions: Patients with freezing may be dissociable based on underlying neurobiological underpinnings which would have significant implications for targeting future treatments. 


\section{Introduction}

Freezing of gait (FOG) remains one of the most poorly treated symptoms of Parkinson's disease (PD) with a devastating impact on quality of life (1). Clinical observations have noted that different situations provoke FOG in different patients (2), which has led clinicians and researchers to question whether multiple subtypes of freezing may exist (3). Here, we examined whether specific environmental triggers, in conjunction with dopaminergic responsiveness might distinguish 'subtypes' of freezers who underwent detailed neurological phenotyping. The ability to accurately distinguish and appropriately identify an individuals' freezing 'subtype' provides the first step towards a much-needed, evidence-based opportunity to tailor management and allow more targeted and individualized intervention.

\section{Methods}

\section{Participants}

Forty-one PD patients with confirmed FOG participated in this study and completed the 'Characterizing Freezing of Gait' (C-FOG) questionnaire (see Supplementary Materials for detailed description), along with the FOG Questionnaire (FOG-Q) (4), the Parkinson's Anxiety Scale (5), Mini-Mental State Exam (6) and the Trail Making Test Parts A and B (Table 1). MDS-UPDRS Part-III (7) was assessed in both the ON and OFF dopaminergic state. Additionally, motor asymmetry was also calculated from items 3.4-3.8, by subtracting the sum of the right items from the sum of the left 
(OFF state). This study was approved by Ethics Board at the University of Sydney, and all participants provided written informed consent

\section{Procedures}

Gait was assessed using eight walking trials (8) (see Figure 1A) during both the OFF- (after a minimum of 12 hours withdrawal) and ON-dopaminergic state. The order of testing was counterbalanced across subjects. All walking trials were video recorded and randomly distributed amongst six independent scorers (MJG, JMH, AJM, MG, JYYS, and KEM). FOG was defined as any point when a participant made a paroxysmal cessation of normal progress (9). The percentage of time spent frozen (\%FOG) was calculated for each trial. Inter-rater variability strong across all conditions (Cronbach's $\alpha=0.980)$.

\section{Statistics and Results}

To assess the concurrent validity of the C-FOG, we correlated the C-FOG items 1.1 (i.e. How often do you experience freezing of gait) and 2.0 (i.e. How long do your freezing of gait episodes typically last?) with the total score from the FOG-Q (4). To determine construct validity, C-FOG items 1.1 and 2.0 were correlated with \%FOG. Significant associations were found between C-FOG item 1.1 and question 3 of the FOG-Q $(r=0.47, p=0.011)$, the FOG-Q total $(r=0.4, p=0.041)$, and the \%FOG in the OFF state $(r=0.41, \quad p=0.019)$, indicating concurrent and construct validity. Furthermore, responses on C-FOG item 2.0 were also significantly correlated with $\%$ FOG in the OFF state $(r=0.54, p=0.002)$. Finally, for the 12 items examining the 
common situations that provoke FOG, Cronbach's $\alpha$ was 0.937 , indicating internal consistency.

\section{Clustering Analysis}

We utilized a data-driven approach to identify clusters that putatively represent previously unrecognized freezing 'subtypes'. To demonstrated the utility of this approach, we next determined whether subjects within separable freezing subtypes were characterized by distinct phenotypic measures across motor, affective and cognitive domains. To this end, de-meaned responses on Section II were correlated with one another using Spearman's rho (Figure 1C). From this matrix, a weightedand signed- version of the Louvain algorithm $(\gamma=1)$ was used to cluster these twelve items (10). A three-cluster solution was associated with a modularity statistic ('Q') of 0.805 , indicating marked community structure within the data (see Figure $1 \mathrm{C}$ and Supplementary Table e1). To further characterize these clusters, we calculated an average de-meaned sub-score for each of the three clusters in each participant. Spearman's correlations were carried out to examine the relationship between each cluster sub-score and participants' clinical phenotype, including \%FOG and FOG-Q total. A $k$-means analysis ( $k=3 ; 100$ iterations) was performed to classify each participant into one of three freezer subgroups, based on their three cluster subscores (the value of $k$ was chosen to match the dimensionality found by the Louvain algorithm). Demographic group differences were examined between the three freezer subgroups. Finally, group differences between the clustered sub-scores were 
compared using independent t-tests that were planned a priori, and clustered subscores were compared within groups using paired samples t-test. Due to the exploratory nature of this study, multiple comparisons corrections were not employed.

\section{Results}

Distinct associations between each of the clusters and the demographic outcomes were identified (Figure 1D). Although Cluster 1 had a significant negative association with motor symptom severity in both the dopaminergic 'ON' ( $r=-0.41)$ and 'OFF' $(r=-$ 0.37) states, Cluster 1 also showed a significant positive association with motor asymmetry $(r=0.34)$, which revealed that greater left motor symptomology was associated with freezing in doorways, while turning, and initiating gait. In contrast, Cluster 2 demonstrated a significant positive correlation with anxiety scores $(r=0.41)$, whilst Cluster 3 had a significant relationship with attentional set-shifting (r-0.42). All clusters were also positively related to freezing severity measured by the percentage of time spent frozen (C1: $r=0.60 ; C 2: r=0.43 ; C 3: r=0.42)$. Given the dissociable relationships between each cluster and the common characteristics across the items within each cluster, we hereafter refer to the three freezer subgroups according to the related phenotype (i.e. Group 1: Asymmetric-Motor; Group 2: Anxious; Group 3: Sensory-Attention). 
Group 1 demonstrated less severe motor symptoms in the OFF state compared to Group $2\left(F_{2,35}=3.64, p=0.035\right)$, and had a greater proportion of PD patients with asymmetric rather than bilateral motor impairment compared Group $2\left(x^{2}=5.8\right.$, $\mathrm{p}=0.016)$ and compared to Group $3\left(x^{2}=5.85, \mathrm{p}=0.016\right)$. There were no other statistical differences between the subgroups (Table 1).

Group 2 had the greatest score on the anxious-related items compared to Group 1 $\left(\mathrm{t}_{26}=-2.19, \mathrm{p}=0.038\right)$ and Group $3\left(\mathrm{t}_{26}=-1.81, \mathrm{p}=0.082\right)$ (Figure 2E). Additionally, Group 3 had the greatest score on the set-shifting-related items compared to Group $1\left(\mathrm{t}_{24}=4.12, \mathrm{p}<0.001\right)$ and Group $2\left(\mathrm{t}_{26}=2.23, \mathrm{p}=0.035\right)$. There were no statistical differences between any of the subgroups on the motor-related items.

Many significant differences were identified when the effects of different situations within each subgroup were compared. Freezers in Group 1 reported most commonly experiencing freezing on 'motor-related items' (e.g. initiating gait, turning and walking through doorways), and their average score on the motor-related items was significantly greater than both the anxious-related items $\left(t_{12}=4.12, p=0.001\right)$ and the set-shifting-related items $\left(t_{12}=7.2, p<0.001\right)$. Group 1 also reported higher scores on average for the anxious-related items (e.g. when rushed, anxious, and distracted) compared to the set-shifting-related items (e.g. walking in the dark, clutter or on a slope; $\left.\mathrm{t}_{12}=2.9, \mathrm{p}=0.034\right)$. Freezers in Group 2 reported most commonly experiencing freezing on 'anxiety-related items' compared to both the motor-related items $\left(\mathrm{t}_{14}=-\right.$ 
3.91, $\mathrm{p}=0.002)$ and the set-shifting-related items $\left(\mathrm{t}_{14}=4.34, \mathrm{p}=0.001\right)$. Finally, freezers in Group 3 reported most commonly experiencing freezing on 'set-shifting-related items' compared to both the motor- and anxious- related items $\left(\mathrm{t}_{12}=-3.67\right.$, $p=003) ;\left(t_{12}=-5.5, p<0.001\right)$. Group 2 also reported higher scores on average for the motor-related items compared to the anxious-related items $\left(t_{12}=2.5, p=0.028\right)$.

Due to the smaller cohort with WALKING assessments, bootstrapping with replacement (100 samples) was used to estimate confidence intervals within each WALKING condition (reported in Supplementary Tables e2 and e3). When collapsed across WALKING conditions, both Group 1 and 3 displayed worse FOG in their OFFstate (i.e. increased average \% time spent frozen), which was substantially reduced when tested in their ON-state (Figure 2F), whereas this pattern was not present in freezers from Group 2.

In the ON state (Figure $2 \mathrm{H}$ ), Group 1 demonstrated the majority of their freezing during the walking trials with a $540^{\circ}$ turn, and substantially less freezing whilst performing the walking trials with dual-task or $180^{\circ}$ turn. Group 2 demonstrated a similar amount of freezing across all three walking conditions $\left(540^{\circ}\right.$ turns, dual-task, and Box Shuffle) compared to $180^{\circ}$. Finally, Group 3 demonstrated most of their FOG whilst performing the dual-task with substantially less freezing across the other three conditions. 
In the OFF state, the pattern of freezing amongst the different subtypes of freezers changed (Figure 2G). Overall, walking around a tight square (i.e. Box Shuffle) became one of the most provocative conditions across all the subgroups of freezers. Group 1 experienced substantially more freezing when performing the dual-task walking trial, whilst Group 3 experienced substantially more freezing when performing the walking trial with a $540^{\circ}$ turn. Notably, freezers in Group 3 experienced much less freezing in the OFF state during the dual-task, however four participants from this subgroup could not complete their walking assessments in the OFF state due to their severity of their freezing.

\section{Discussion}

Here, we introduce the C-FOG questionnaire as a promising instrument for detecting and classifying subtypes of freezers. Unlike other freezing questionnaires, the CFOG provides novel insights into the heterogeneity inherent to freezing and the situations that trigger this enigmatic phenomenon. Together, these results provide preliminary evidence for distinct Asymmetric-Motor, Anxious, and Sensory-Attention phenotypes within FOG. This heretofore unrecognized heterogeneity may underlie known inconsistencies in prior empirical literature (3).

The freezing phenotypes identified in this study putatively represent cohorts with distinct 'upstream' dysfunctions, in which idiosyncratic pathophysiological mechanisms overwhelm specialized neural circuitry unique to each phenotypic subtype, which then ultimately manifests via a common inhibitory brainstem pathway 
that arrests ongoing gait processes $(11,12)$. This interpretation predicts that different subtypes of patients with FOG should each demonstrate unique susceptibility to situations that provoke freezing, which should in turn be directly linked to the particular domains that relate to their phenotypic expression. For example, if one is highly anxious, the incoming input from the limbic system to the striatum could overload the processing capacity of the gait system, leading to FOG. In contrast, an individual with impaired motor automaticity (and hence, an over-reliance on the cognitive control of gait), may fall victim to instances that perturb or divide cognitive resources. However, in each case, the 'final common pathway' may indeed be shared. Future studies are now required to disambiguate these alternatives.

To provide true clinical utility, our study should be replicated in an expanded cohort, with the identified clusters used as statistical priors. Further multi-centre research is also needed to determine whether these findings are reproducible and reliable. Future studies should also carefully consider the type of dopaminergic treatments to determine whether therapeutic patterns contribute to the FOG phenotypes observed in this preliminary study. Nonetheless, the proposition of different subtypes of freezing has important clinical implications for individualized and targeted treatment strategies. 
Contributions: Kaylena A. Ehgoetz Martens - conception, assisted with construction of the C-FOG questionnaire, data collection, data processing, data analysis, drafting manuscript; James M. Shine - conception, assisted with construction of the C-FOG questionnaire, data collection, data analysis, review and revision of manuscript; Courtney C. Walton - assisted with construction of the CFOG questionnaire, data collection, review and revision of manuscript; Matthew J. Georgiades - data collection, data processing, review and revision of manuscript; Moran Gilat - assisted with construction of the C-FOG questionnaire, data collection, review and revision of manuscript; Julie M. Hall - data collection, data processing, review and revision of manuscript; Alana Muller - data collection, data processing; Jennifer Szeto - data collection, data processing, review and revision of manuscript; Simon J.G. Lewis - conception, review and revision of manuscript

\section{References}

1. Walton CC, Shine JM, Hall JM, O'Callaghan C, Mowszowski L, Gilat M, et al. The major impact of freezing of gait on quality of life in Parkinson's disease. J Neurol. Springer Berlin Heidelberg; 2015 Jan;262(1):108-15.

2. Nutt JG, Bloem BR, Giladi N, Hallett M, Horak FB, Nieuwboer A. Freezing of gait: Moving forward on a mysterious clinical phenomenon. Lancet Neurol [Internet]. Elsevier Ltd; 2011;10(8):734-44. Available from: http://dx.doi.org/10.1016/S1474-4422(11)70143-0

3. Fasano A, Herman T, Tessitore A, Strafella AP, Bohnen NI. Neuroimaging of 
freezing of gait. J Parkinsons Dis. 2015;5(2):241-54.

4. Giladi N, Shabtai H, Simon ES, Biran S, Tal J, Korczyn AD. Construction of freezing of gait questionnaire for patients with Parkinsonism. Park Relat Disord. 2000;6(3):165-70.

5. Leentjens AFG, Dujardin K, Pontone GM, Starkstein S, Weintraub D, MartinezMartin P. The Parkinson Anxiety Scale (PAS): development and validation of a new anxiety scale. Mov Disord. 2014;29(8):1035-43.

6. Folstein MF, Folstein SE, Mchugh PR. "Mini-mental state": A Practical method for grading the cognitive state of patients for the clinician. J Psychiatr Res. Pergamon Press; 1975;12(3):189-98.

7. Goetz CG, Fahn S, Martinez-Martin P, Poewe W, Sampaio C, Stebbins GT, et al. Movement Disorder Society-sponsored revision of the Unified Parkinson's Disease Rating Scale (MDS-UPDRS): Process, format, and clinimetric testing plan. Mov Disord [Internet]. 2007 Jan [cited 2014 Oct 7];22(1):41-7. Available from: http://www.ncbi.nlm.nih.gov/pubmed/17115387

8. Shine JM, Moore ST, Bolitho SJ, Morris TR, Dilda V, Naismith SL, et al. Assessing the utility of Freezing of Gait Questionnaires in Parkinson's Disease. Park Relat Disord [Internet]. Elsevier Ltd; 2012;18(1):25-9. Available from: http://dx.doi.org/10.1016/j.parkreldis.2011.08.002

9. Schaafsma JD, Balash Y, Gurevich T, Bartels AL, Hausdorff JM, Giladi N. Characterization of freezing of gait subtypes and the response of each to levodopa in Parkinson's disease. Eur J Neurol [Internet]. 2003 Jul;10(4):391-8. 
Available from: http://doi.wiley.com/10.1046/j.1468-1331.2003.00611.x

10. Blondel VD, Guillaume J-L, Lambiotte R, Lefebvre E. Fast unfolding of communities in large networks. J Stat Mech Theory Exp. 2008;2008.

11. Snijders AH, Takakusaki K, Debu B, Lozano AM, Krishna V, Fasano A, et al. Physiology of freezing of gait. Ann Neurol. 2016;80(5):644-59.

12. Lewis SJG, Shine JM. The Next Step: A Common Neural Mechanism for Freezing of Gait. Neurosci [Internet]. 2014;1073858414559101-. Available from:

http://nro.sagepub.com/cgi/doi/10.1177/1073858414559101\%5Cnhttp://nro.sa gepub.com/content/early/2014/11/14/1073858414559101.long

13. Ehgoetz Martens KA, Hall JM, Gilat M, Georgiades MJ, Walton CC, Lewis SJG. Anxiety is associated with freezing of gait and attentional set-shifting in Parkinson's disease: A new perspective for early intervention. Gait Posture [Internet]. Elsevier B.V.; 2016;49:431-6. Available from: http://dx.doi.org/10.1016/j.gaitpost.2016.07.182

14. Walton CC, Shine JM, Mowszowski L, Naismith SL, Lewis SJG. Freezing of gait in Parkinson's disease: Current treatments and the potential role for cognitive training. Restor Neurol Neurosci. IOS Press; 2014;32(3):411-22. 


\section{Figure Captions}

Figure 1. (A) depicts the modified timed gait assessment protocol; (B) shows the relationship between the C-FOG Section II total and the average percentage of time spent frozen during the timed gait assessments; (C) displays the similarity matrix of the C-FOG Section II items ordered based on the Louvain clustering solution (which is also reported in Supplementary Material); (D) depicts the correlation coefficient for each cluster and its' relationship to motor asymmetry (bradykinesia), the score on the Parkinson's Anxiety Scale, and the performance on the Trail Making Task Part B; (E) shows the average sub-score for each group on the Section II of the C-FOG; (F) illustrates the effect of dopaminergic medication on the percent of time spent frozen during the timed gait assessment across each group; (G) displays the effect of condition during the timed gait assessment on the percentage of time spent frozen in the OFF dopaminergic state across each group of freezers; $(\mathrm{H})$ displays the effect of 
each condition on the percentage of time spent frozen in the $\mathrm{ON}$ dopaminergic state across groups.

This article is protected by copyright. All rights reserved. 


\section{University Library}

\section{- M M N E R VA A gateway to Melbourne's research publications}

Minerva Access is the Institutional Repository of The University of Melbourne

\section{Author/s:}

Martens, KAE;Shine, JM;Walton, CC;Georgiades, MJ;Gilat, M;Hall, JM;Muller, AJ;Szeto, JYY;Lewis, SJG

Title:

Evidence for Subtypes of Freezing of Gait in Parkinson's Disease

\section{Date:}

2018-07-01

\section{Citation:}

Martens, K. A. E., Shine, J. M., Walton, C. C., Georgiades, M. J., Gilat, M., Hall, J. M., Muller, A. J., Szeto, J. Y. Y. \& Lewis, S. J. G. (2018). Evidence for Subtypes of Freezing of Gait in Parkinson's Disease. MOVEMENT DISORDERS, 33 (7), pp.1174-1178. https:// doi.org/10.1002/mds.27417.

Persistent Link:

http://hdl.handle.net/11343/261104 